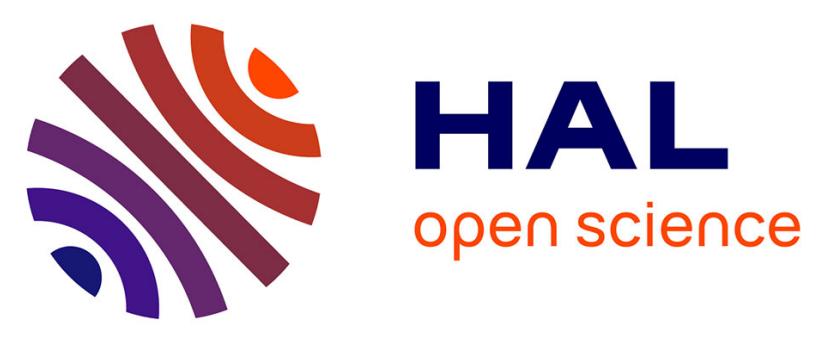

\title{
Scaling strength distributions in quasi-brittle materials from micro- to macro-scales: A computational approach to modeling Nature-inspired structural ceramics
}

Martin Genet, Guillaume Couégnat, Antoni P. Tomsia, Robert O. Ritchie

\section{- To cite this version:}

Martin Genet, Guillaume Couégnat, Antoni P. Tomsia, Robert O. Ritchie. Scaling strength distributions in quasi-brittle materials from micro- to macro-scales: A computational approach to modeling Nature-inspired structural ceramics. Journal of the Mechanics and Physics of Solids, 2014, 68, pp.13. 10.1016/j.jmps.2014.03.011 . hal-01196360

\section{HAL Id: hal-01196360 \\ https://hal.science/hal-01196360}

Submitted on 9 Sep 2015

HAL is a multi-disciplinary open access archive for the deposit and dissemination of scientific research documents, whether they are published or not. The documents may come from teaching and research institutions in France or abroad, or from public or private research centers.
L'archive ouverte pluridisciplinaire HAL, est destinée au dépôt et à la diffusion de documents scientifiques de niveau recherche, publiés ou non, émanant des établissements d'enseignement et de recherche français ou étrangers, des laboratoires publics ou privés. 


\title{
Scaling strength distributions in quasi-brittle materials from micro to macro scales: A computational approach to modeling Nature-inspired structural ceramics
}

\author{
Martin Genet ${ }^{\mathrm{a}, \mathrm{b}, *}$, Guillaume Couégnat ${ }^{\mathrm{c}}$, Antoni P. Tomsia ${ }^{\mathrm{a}}$, Robert O. \\ Ritchie $^{\mathrm{a}, \mathrm{d}}$ \\ ${ }^{a}$ Materials Sciences Division, Lawrence Berkeley National Laboratory, California, USA \\ ${ }^{b}$ Marie-Curie International Outgoing Fellow, Surgery Department, University of \\ California at San Francisco, USA \\ ${ }^{c}$ Laboratoire des Composites Thermostructuraux (CNRS - Univ. Bordeaux - Herakles - \\ CEA), Pessac, France \\ ${ }^{d}$ Department of Materials Science and Engineering, University of California at Berkeley, \\ $U S A$
}

\begin{abstract}
This paper presents an approach to predict the strength distribution of quasi-brittle materials across multiple length-scales, with emphasis on Nature-inspired ceramic structures. It permits the computation of the failure probability of any structure under any mechanical load, solely based on considerations of the microstructure and its failure properties by naturally incorporating the statistical and size-dependent aspects of failure. We overcome the intrinsic limitations of single periodic unit-based approaches by computing the successive failures of the material components and associated stress redistributions on arbitrary numbers of periodic units. For large size samples, the microscopic cells are replaced by an homogenized continuum with equivalent stochastic and damaged constitutive behavior. After establishing the predictive capabilities of the method, and illustrating
\end{abstract}

\footnotetext{
${ }^{*}$ Corresponding author. Mail address: Lawrence Berkeley National Laboratory, One Cyclotron Road MS62-0237, Berkeley, CA-94720, USA. Tel: 1-510-486-6809. E-mail address: mgenet@lbl.gov.
} 
its potential relevance to several engineering problems, we employ it in the study of the shape and scaling of strength distributions across differing length-scales for a particular quasi-brittle system. We find that the strength distributions display a Weibull form for samples of size approaching the periodic unit; however, these distributions become closer to normal with further increase in sample size before finally reverting to a Weibull form for macroscopic sized samples. In terms of scaling, we find that weakest link scaling applies only to microscopic, and not macroscopic scale, samples. These findings are discussed in relation to failure patterns computed at different size-scales.

\section{Highlights:}

- Analysis of quasi-brittle failure including statistical and size-dependent aspects

- Use of computational homogenization to compute up to macroscopic scale samples

- Application to Nature-inspired ceramic structures made by freezecasting

- Strength distribution shape converges to Weibull for macroscopic scale samples

- Weakest-link scaling does not apply to the Weibull-like macroscopic strength distributions

Keywords:

Fracture; Microcracking; Ceramics; Finite element analysis; Computational homogenization 


\section{Contents}

1 Background and Significance $\quad 3$

2 Modeling and Methods $\quad 8$

2.1 The reference micromechanical model . . . . . . . . . . 8

2.2 Computational homogenization-based scaling method for strength distribution . . . . . . . . . . . . 12

3 Results and Applications $\quad 15$

3.1 Response of the reference micromechanical model . . . . . . . 15

3.2 Limitations of the weakest link theory . . . . . . . . . . . 18

3.3 Validation of the computational homogenization-based scaling method for strength distribution . . . . . . . . . . 22

3.4 Application to failure prediction of macroscopic scale samples 22

4 Summary and Perspectives

\section{1. Background and Significance}

2 Many materials exhibit a quasi-brittle behavior, i.e., their ultimate fail3 ure is triggered by a significant number of local events (in contrast to the 4 purely brittle behavior of many ceramics and glasses), yet still is not pre5 ceded by highly dissipative processes associated with large inelastic de6 formations and strain hardening (as with ductile materials like metals).

7 Such behavior is found in geological (e.g., rocks), biological (e.g., bone) 8 and engineering/constructional (e.g., ceramic composites, concrete) mate9 rials [Bažant, 1999, 2004]. In this paper, we are particularly interested in 10 cellular ceramic structures, which have recently found potential high-impact 
1 applications in tissue engineering [Deville et al., 2006] and high-performance 2 composites [Munch et al., 2008].

3 One issue with the engineering use of quasi-brittle materials is associated 4 with the statistical and size-dependence of their failure properties, which 5 can make reliable predictions a difficult challenge. Experimental analysis 6 are often of little help as they cannot reach the target failure probabilities 7 required for certification; for example, a prescribed failure probability of $810^{-6}$ would require $10^{6}$ repeated experiments. Moreover, the standard 9 procedures of fracture mechanics, consisting of studying smaller scale samples and then extrapolating the results to larger, more realistically scaled

11 samples, are limited by the lack of methods which are effectively able to 12 "bridge the length-scales". Thus, for material design and failure prediction 13 of quasi-brittle materials for engineering applications, experimental studies 14 must be augmented by theoretical tools based on mechanical modeling. 15 However, failure can be a complex phenomenon to model, as it involves 16 both local and global phenomena, i.e., small defects induce localized cracks 17 and stress redistribution at nano- to micro-scales coupled with the fact that the macro-scale size of a structure can statistically dictate the probability 9 of activating the worse-case defects.

Many authors have studied the failure of quasi-brittle materials, from such multiple viewpoints. Our objective here is not to draw an exhaustive portrait of the field, but to note several pertinent studies to better position our own approach. A critical analysis is undoubtedly the Weibull theory which describes the failure of brittle ("in-series") systems, based on a specific strength (i.e., Weibull) distribution and a power law for the volumetric scaling [Weibull, 1939, 1951; Hild, 2001], together with that of Daniels who 
established that the corresponding strength distribution of large in-parallel systems must tend toward a Gaussian distribution [Daniels, 1945]. These analyses are essential for the understanding of the statistical failure of quasi4 brittle materials. They have been extended by many authors to account 5 for, e.g., multiaxial fracture in Weibull theory [Evans, 1978; Guillaumat 6 and Lamon, 1996], or different load sharing mechanisms in Daniels theory 7 [Phoenix, 1974, 1978; Calard and Lamon, 2004]. They are, however, limited 8 in their application to realistic systems, as for example with Daniels theory 9 which fails to describe the transition from Weibull to Gaussian behavior, and to predict the distribution's tail (which cannot be Gaussian) [Bažant, 2004; Bažant and Pang, 2007].

12 With respect to cellular ceramics, Gibson \& Ashby [Gibson and Ashby, 1997] derived the structure-stiffness relationships for many porous structures simply using beam and plate theories, although their approach cannot 5 directly treat the statistical and size-dependent aspects of failure.

16 Similar micromechanical approaches have been proposed for many bio17 logical and synthetic quasi-brittle materials, e.g., [Ji and Gao, 2004; Begley 18 et al., 2012], but again the key stochastic and size-dependent aspects of 19 quasi-brittle failure were not directly considered. (These analyses are invariably based on a single representative volume element, where Cox's shear lag principle [Cox, 1952] is used to estimate the redistribution of stresses around cracks.)

There are also the purely macroscopic approaches, e.g., [De Borst et al., 1995; Desmorat et al., 2007; Genet et al., 2013b]; but as these analyses are based on continuum damage mechanics [Lemaître and Desmorat, 2005;

26 Lemaître et al., 2009], they cannot explicitly model microstructure or micro27 scopic damage processes, but only their indirect effect on the macroscopic 
1 mechanical properties. They are, however, extremely efficient at dealing 2 with specific structures and loads, but require a large amount experimental data for calibration, and are not suitable to derive true structure-properties relationships.

5 An intermediate approach is that of Bažant et al. [Bažant et al., 1991;

6 Bažant and Xi, 1991; Bažant, 1999, 2004]. Based on energetic principles, 7 these authors were able to derive scaling laws for the strength of various 8 quasi-brittle materials, although this method does not permit the scaling of 9 the distributions themselves [Bažant, 2004]. More recently, they introduced a hierarchical model of chains and bundles of representative volume ele11 ments (RVEs), starting from the atomic scale, to derive some fundamental 12 conclusions on the theoritical scaling of strength in quasi-brittle systems 13 [Bažant and Pang, 2007; Bažant et al., 2009; Le et al., 2011; Le and Bažant, 14 2011]. Most importantly, they were able to predict the transition from 15 Gaussian to Weibull of the strength distributions of structures of increasing 16 sizes [Bažant and Pang, 2007].

17

In a recent article, we presented our first approach to bridge the scales, 19 with a model based on Sanchez-Palencia's theory of periodic homogeniza20 tion and Weibull's theory of statistical failure [Genet et al., 2013a], with 21 application to robocast scaffolds [Houmard et al., 2013]. Material struc22 ture is introduced at microscopic scales, while the sample size is naturally 23 handled on the macroscopic level, the two dimensions being linked through 24 homogenization; statistical failure is then predicted through the computa25 tion of a Weibull-like integral at both size-scales. This approach has sig26 nificant predictive capabilities but also limitations; as the successive failure 27 of the material's constituents are not explicitly represented, a virtual, ad 
1 hoc, "macroscopic" crack population is introduced, which must be identified 2 experimentally on the macroscopic scale.

3 In the present paper, we propose a computational method to directly 4 link the strength distributions of the constituents of quasi-brittle materi5 als and macroscopic samples made from these constituents. The idea is 6 to overcome the intrinsic limitations of approaches based on a single RVE, 7 which are really only suitable to deal with homogeneous phenomena (on the 8 scale of the structure), but not strictly with localized events such as those triggering failure. We achieve this by modeling as many RVEs as necessary to produce reliable predictions. Since the number of RVEs that can 11 be modeled at a microscopic level of description is rapidly limited by com12 putational capabilities, we introduce a multi-level numerical method which 13 permits the computation of samples of virtually any size, with essentially no loss of information compared to a direct microscopic computation but with 15 a drastically reduced computational cost. Micro-cells, where physical mech-

anisms are finely described, are replaced by mechanically and statistically equivalent "macro-cells" containing only a very few degrees of freedom. As a consequence, structural-level computations can be run at a very reduced cost, and a large number of stochastic cases can be explored in a reasonable time.

Fundamentally, we build upon [Bažant and Pang, 2007; Bažant et al., 2009; Le et al., 2011; Le and Bažant, 2011] and study the scaling of strength induced by both the intrinsic micro-scale defects and the ones generated by the microstructure itself, i.e., the stress redistribution induced by its geometrical features. An important difference with these previous works is that we do not need to idealize the considered microstructure as a series of chains and bundles since we perform direct numerical computations on the 
1 real microstructure. Thus, stress redistributions are directly induced by the

2 laws of continuum mechanics and the features of the studied microstructure itself, without any additional assumptions.

4 Our approach is general, and can be applied to any cellular ceramic, 5 indeed to any quasi-brittle material. We illustrate the methodology here 6 with reference to the ceramic scaffolds that can be made by freeze-casting 7 [Deville et al., 2006; Munch et al., 2008; Naglieri et al., 2013]. In order to 8 focus on the method itself, which is presented section 2.2, we first develop 9 a simple micromechanical model (section 2.1), and then present some key results, including numerical validation (section 3.3), comparison to a basic 11 power law ("in-series") scaling (section 3.2), and application to macro-scale 12 samples (section 3.4).

\section{Modeling and Methods}

\subsection{The reference micromechanical model}

Our approach in this paper is on the failure prediction of porous ceramic scaffolds made by freeze-casting [Deville et al., 2006; Munch et al., 2008; Naglieri et al., 2013]. A scanning electron microscopy (SEM) image of a scaffold is shown Figure 1(a); our associated idealized geometry in Figure 1(b). This geometry consists of a lamellar ceramic framework linked by periodic bridges to give a brick-like structure, which resembles a coarse nacre-like architecture; in the final bio-inspired materials, the pores in between the "bricks" are infiltrated with a compliant phase, i.e., a polymer or metal, to give a highly damage-tolerant "brick-and-mortar" structure [Munch et al., 2008]. To focus on the theoretical strategy itself, we have restricted the analysis to in-plane properties, and chosen a simple geometry, 
perfectly periodic and deterministic, characterized by only three parameters, namely the distances between brick walls and bridges, respectively, $d_{w}$ and $d_{b}$, and the thickness, $e$, of these walls and bridges (Figure 1(b)). An additional parameter must also be introduced to fully define the computed microcells, namely the number, $r$, of RVEs that they contain. Note that several authors have proposed methods to generate statistical microstructures from images such as the one in Figure 1(a) [Jeulin, 2001; Torquato, 2002; Couégnat, 2008], although this has not been undertaken in the present model.

With respect to the phenomenology, the macroscopic failure of these cellular ceramics is induced by the successive failures of individual constitutive walls. Such local failures are triggered by the activation of small defects in tension or shear, or by the wall bending in compression. The failures are highly probabilistic because the distributions of sizes and shapes of defects and walls are very broad. There is other important process that appears in compression, that of the crushing of broken walls, which ultimately results in the ceramic scaffold becoming fully fragmented; for the sake of simplicity we do not consider wall bending/crushing in compression in the current variant of the model. (Such crushing in cellular ceramics usually occurs beyond the scope of application of most models, as the material is then fully fragmented and cannot withstand any other load than compression.)

Thus, initially the micromechanical model will only be developed to consider the defect-activated failure of the ceramic walls, which are assumed to display isotropic elastic-brittle behavior with Young's modulus $E$ and Poisson's ratio $\nu$. The response of the microstructure to mechanical loading is computed using the finite element method. Since the defects are actually too small and too numerous to be characterized, Weibull theory [Weibull, 
1 1939] will be used here to model the wall failures. ${ }^{1}$

As the Weibull theory is a non-local theory of fracture, and we need to represent the successive failures of walls and bridges, they must be split between several elements of volume. This decomposition depends on the considered microstructure, and is illustrated for the freeze-cast scaffold in Figure 1(b), where every color represents a single element of volume (note the periodicity of the border volume elements). Basically, every bridge is an element of volume, as well as every piece of wall between two bridges. For each element of volume, it is assumed that failure is triggered by positive deformations, a hypothesis often made for brittle and quasi-brittle materials [Mazars and Pijaudier-Cabot, 1989; Lemaître and Desmorat, 2005; Genet et al., 2012; Fagiano et al., 2014], and incorporated in the Weibull framework in [Genet et al., 2013a]. The failure probability of any given element of volume is then:

$$
\begin{aligned}
p^{F} & =1-\exp \left(-\frac{V}{V_{0}}\left(\frac{\widetilde{\epsilon}}{\epsilon_{0}}\right)^{m}\right) \\
\text { with } \tilde{\epsilon} & =\frac{1}{V} \int_{V}\left\|\langle\underline{\underline{\epsilon}}\rangle_{+}\right\| d V
\end{aligned}
$$

2 where $V$ is its volume and $V_{0}$ a reference volume, $\underline{\underline{\epsilon}}$ is the strain tensor 3 field, $\langle\cdot\rangle_{+}$denotes the positive part of second order symmetric tensors in the 4 classical sense [Lemaître et al., 2009], and $\epsilon_{0}$ and $m$ are, respectively, the 5 two classical Weibull coefficients [Weibull, 1939; Hild, 1998].

$6 \quad$ Each element of volume contains a potential crack, which is initially

\footnotetext{
${ }^{1}$ Despite the fact that it was introduced by Weibull himself based on phenomenological considerations [Weibull, 1939], it was later proven to have more fundamental basis; the theory actually relies upon a Poisson's distribution of defect sizes and a simple fracture criterion [Freudenthal, 1968; Hild, 1998; Bažant, 1999]. Note that more complex fracture criteria can be used, leading to slightly different laws [Batdorf and Heinisch, 1978].
} 
4 be positioned at the middle of each element of volume. ${ }^{2}$ At the beginning of

closed but will eventually become opened at some point in the computation. Since the position of the crack within the element of volume is not really significant for the remainder of the computation, such cracks will arbitrarily the computation, every element of volume is given a critical probability of failure, i.e., a random number in the range ]0;1[. During the loading, when the probability of failure of an element of volume reaches its critical value, then it is considered as broken, and the potential crack that it contains is considered open.

This simple model permits the representation of the successive failures of the constituents of a piece of ceramic scaffold of arbitrary size under arbitrary load, from the initial to critical failure event, i.e., from damage initiation to macroscopic crack initiation, and as such provides an assessment of the statistical strength of the scaffold. Note that the model also allows an evaluation of the failure of the walls and bridges at the macroscopic crack tip, i.e., of the propagation of a macroscopic crack, and therefore can provide an assessment of the toughness of the scaffold, although this feature will not be addressed in the present paper.

The geometrical and materials parameters of the freeze-cast ceramic scaffolds used for the computations are presented Tables 1 and 2.

With respect to the computational procedures, we used GMSH [Geuzaine and Remacle, 2009] (coupled with an in-house Python code) to generate (triangular) meshes, and the LMT++ library [Leclerc, 2010; Genet, 2010] (which uses the CHOLMOD linear solver [Chen et al., 2008]) for finite ele-

\footnotetext{
${ }^{2}$ Note that it was already shown for similar computations that choosing a probabilistic position has no significant effect on the model's predictions [Lamon, 2009].
} 


\begin{tabular}{c|c|c}
$d_{w}(\mu m)$ & $d_{b}(\mu m)$ & $e(\mu m)$ \\
\hline 25 & 75 & 5
\end{tabular}

Table 1: Geometrical coefficients used for the computations presented in this paper: $d_{w}$ is the distance between the walls, $d_{b}$ the distance between the bridges, and $e$ the walls and bridges thickness.

1 ment computations.

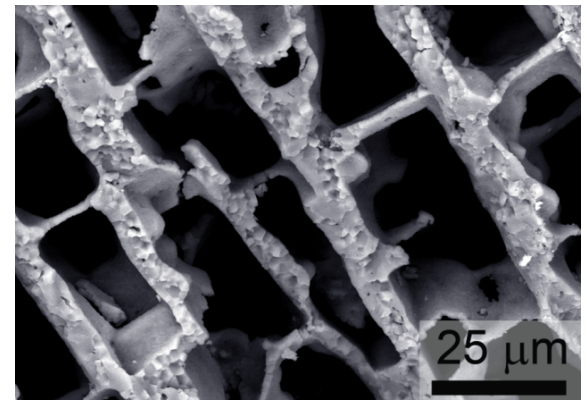

(a) SEM image [Launey et al., 2009]

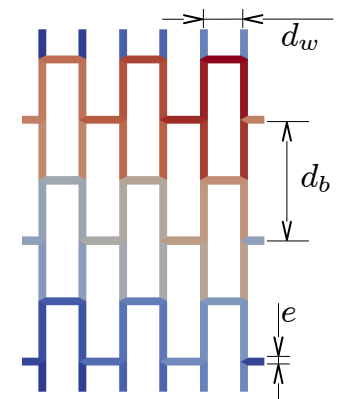

(b) Idealized geometry

Figure 1: Representative SEM image of a ceramic scaffold made by freeze-casting, and its associated idealized geometry. The idealized geometry consists of walls connected by bridges positioned in staggered rows. There are three geometrical parameters: $d_{w}$, the distance between the walls; $d_{b}$, the distance between the bridges; $e$, the thickness of the walls and bridges. A microcell is defined by $r \times r$ RVEs. To describe the successive failures of walls and bridges, the scaffold is divided into many elements of volume, represented here in different colors (note the periodicity of the border volume elements).

2.2. Computational homogenization-based scaling method for strength distribution

There is actually no theoretical way to scale strength distributions of sys-

5 tems with complex failure patterns such as the one presented in the previous

6 section. In this paper, we propose the computational method to achieve this

7 for any quasi-brittle system illustrated in Figure 2. 


\begin{tabular}{c|c|c|c|c}
$E(\mathrm{MPa})$ & $\nu()$ & $V_{0}\left(\mathrm{~mm}^{2}\right)$ & $\sigma_{0}(M P a)$ & $m()$ \\
\hline 3.5 & 0.2 & 1 & 100 & 5
\end{tabular}

Table 2: Material coefficients used for the computations presented in this paper: $E$ and $\nu$ are the Young's modulus and Poisson's ratio of the walls and bridges; $V_{0}, \sigma_{0}$ and $m$ are the three Weibull coefficients (i.e., reference volume, scale parameter and shape parameter) of the walls and bridges. Note that $\epsilon_{0}=\sigma_{0} / E$, in Equation (1).

1 On the macroscopic scale, we have a general continuum mechanics prob2 lem, with a sample submitted to boundary conditions and loading (repre3 sented here by the external traction $T$ ). Since the system is probabilistic, 4 its failure will follow a probability law. The objective of our method is 5 to compute this probability law solely based upon the mechanical proper6 ties of the sample's constitutive material, without additional assumptions 7 or parameters. To achieve this, the macroscopic problem is discretized and 8 solved using the finite element method, where each element is given a size9 dependent and probabilistic mechanical behavior interpolated among a set of responses pre-computed on the microscopic level. It is important to note that the method is not restricted to the study of macroscopically homogeneous systems, but could handle cases, without modification, where, for example, the local material orientation changes from one region to the other, 14 as it is the case for the structure shown in Figure 1(a).

Pre-computations for a given micro-cell (i.e., geometry of the RVE, num16 ber of RVEs, elastic and failure material properties) comprise computing, 17 for a set of macroscopic loading $\underline{\underline{\Sigma}}_{i}$, the micro-cell's range of stochastic responses, as illustrated in Figure 4. A possible set of 2D macroscopic tension load cases is shown in Figure 3, with canonical (i.e., pure tension in each di20 rection, plus pure shear) and intermediate loading directions. As many sets 
1 are possible, it is important to select as many cases as needed for the anal2 ysis. The macroscopic finite element behavior is then interpolated between 3 the pre-computed behaviors. In practice, for a given stress $\triangleq$ applied to an 4 element which does not correspond a priori to any of the pre-computed cases $5 \underline{\underline{\Sigma}}_{i}$, we compute the associated strain as a linear combination of strains asso6 ciated with neighboring load cases, using the same interpolation for stresses 7 and strains. One recognizes here the iso-parametric principle used in finite 8 element technology, where the same shape functions are used to interpolate

both position and displacement from nodes. Because we are presenting only results on unidirectional load cases, for the computations carried out in this work, we have pre-computed solutions for only one loading direction, which corresponds to the macroscopic loading direction.

Resulting size effects on the macroscopic level are then directly handled through a competition between microscale failures and multiscale stress redistribution. Our method allows the computation of the strength distribution of any structure under any loading, solely from the stochastic behavior of its constitutive material. Because of this two-level approach, the computation is achieved at a much lower cost than if run directly based upon the micromodel (which would be impossible for macro-scale samples), with virtually no information loss. Let us also point out that if needed, the method could be extended with more than two levels, so that pre-computations would be run scale by scale, from the micromodel up to the desired structural level. Thus, with enough levels of homogenization, the computational cost of solving structural problems becomes low enough to perform thousands, if not millions, of cases in a reasonnable time.

On a more technical basis, we implemented the multi-level method using an in-house finite element framework [Couégnat et al., 2013] with the 
1 MUMPS library [Amestoy et al., 2000] as a linear solver.

\section{3. Results and Applications}

\section{3.1. Response of the reference micromechanical model}

Figure 4 shows the result of one run of the micromechanical model, previously introduced in Section 2.1, on a portion of scaffold of size $r=5 \times 5$ RVEs under pure traction with periodic boundary conditions. The resulting stress-strain curve is shown, as well as the strain fields over the deformed geometries for several states reached during the computation. It is important to note that, even if its ingredients are relatively basic, the present model is already able to capture several fundamental features of actual failures of the ceramic scaffolds, specifically that: (i) both bridges and wall failures occur, (ii) bridges and wall failures are present outside the main crack, i.e., there is damage away from the macroscopic crack, (iii) the main crack is not fully straight, and not fully orthogonal to the loading direction.

This micromechanical model can be used to compute the strength distributions of micro-cells of virtually any size, under any loading, with virtually any precision; the actual size of the considered micro-cell is evidently limited by the computational cost, hence the interest of the two-level method presented in this paper. Figure 5 represents the cumulative strength distribution of micro-cells of size $r=1 \times 1,2 \times 2,4 \times 4,8 \times 8$ and $16 \times 16$ RVEs, loaded in tension in the direction parallel to the bridges. Strength distributions are defined as follows: for a given series of $\mathrm{N}$ runs, the strength values are sorted in ascending order, and then assigned a failure probability of $1 /(N+1), 2 /(N+1), \ldots, N /(N+1)$. At least 1000 runs were computed for each size, so that the failure probabilities go from $\approx 0.001$ to $\approx 0.999$. 


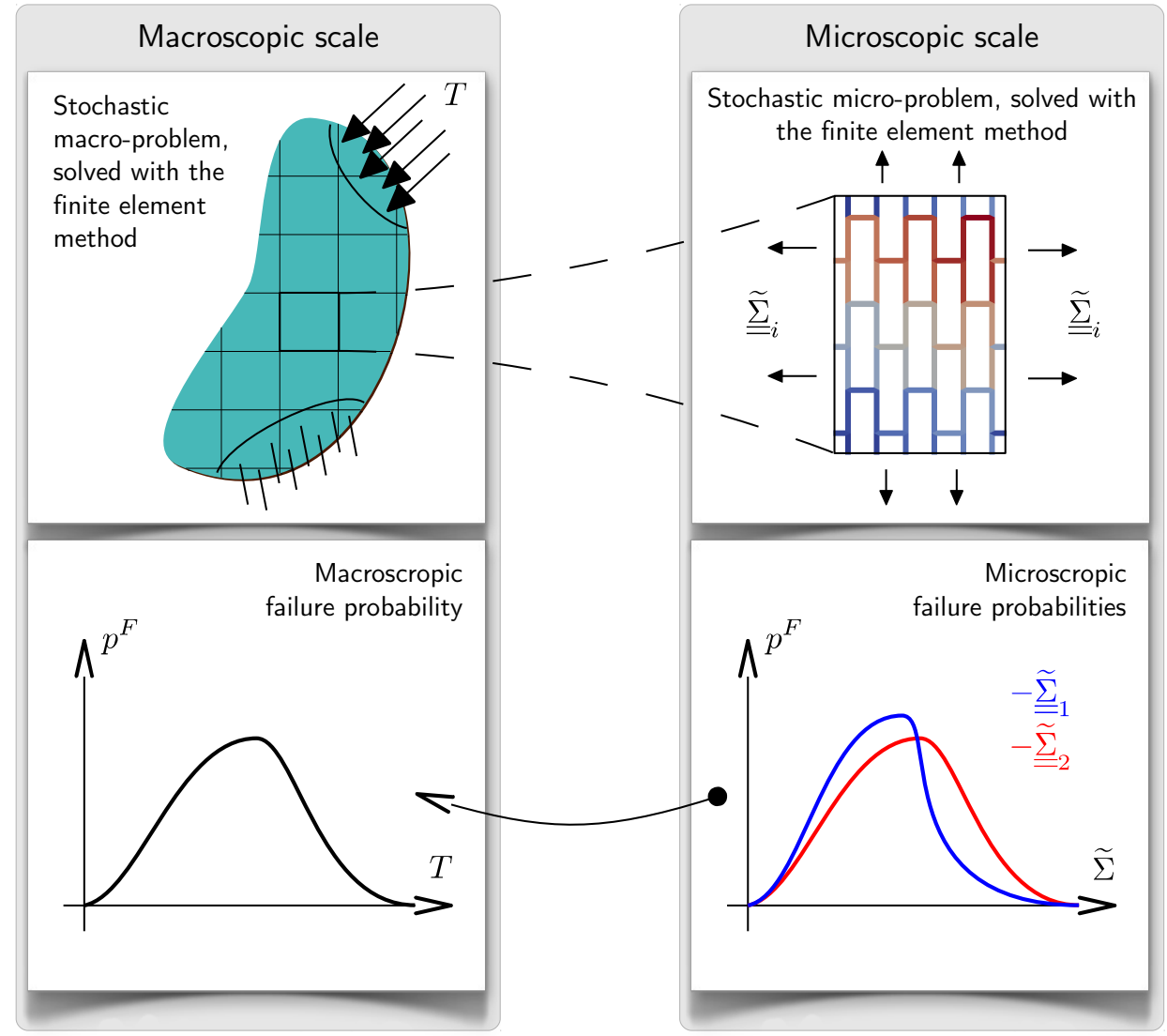

Figure 2: Illustration of our multi-level method. The main goal is to compute the macroscopic failure probability law solely based upon the mechanical properties of the sample's constitutive material on the microscopic level. The macroscopic problem is discretized and solved using the finite element method. Each finite element is given a size-dependent and probabilistic mechanical behavior, interpolated among a set of responses pre-computed on the microscopic level. 

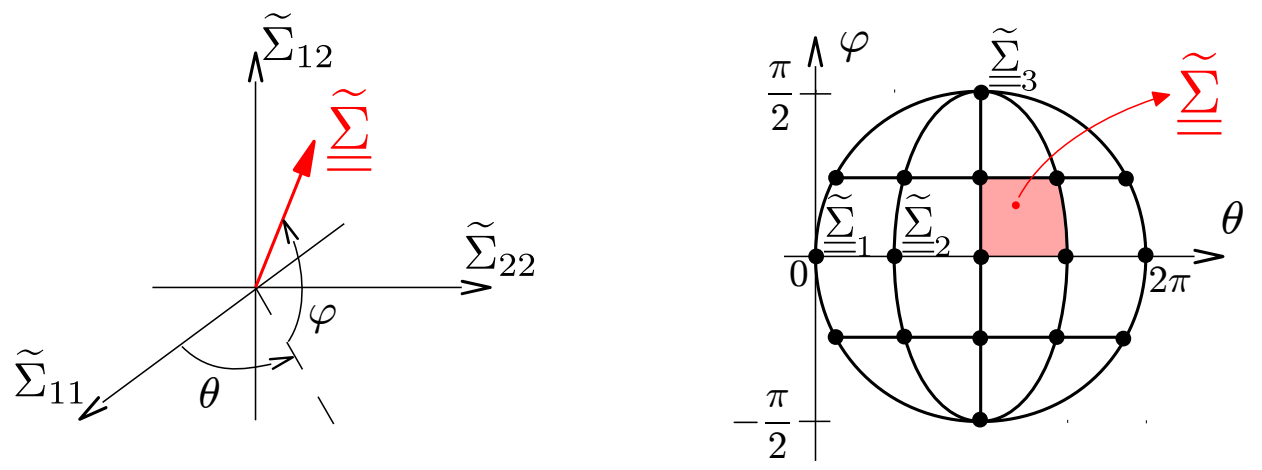

Figure 3: An example of a set of 2D macroscopic loading cases for the stochastic precomputations on the micro-cell, containing the canonical loading cases $\widetilde{\underline{\Sigma}}_{1}$ (pure tension in the bridges direction), $\underline{\underline{\Sigma}}_{2}$ (pure tension in the wall directions) and $\underline{\underline{\Sigma}}_{3}$ (pure shear), as well as intermediate cases. For a given macroscopic finite element stress, the behavior is interpolated between the neighboring pre-computed microscopic behaviors.

1 This figure clearly illustrates the major size effect in this structure, with an 2 average strength reduced by a factor of two between sizes of $r=1 \times 1$ and 3 $r=16 \times 16$.

$4 \quad$ In addition to the stress-strain curve and the strength computed on each 5 run, the evolution of the homogenized elastic properties is also calculated 6 for the homogenized computations described in section 2.2. Thus, for any 7 deformation level, we know the distribution of homogenized stiffness tensors 8 (more precisely, the distributions of their components) of the micro-cells. 9 Figure 6 shows the distributions, specifically the mean value and those at $10 \quad 10 \%$ and $90 \%$, of the components of the homogenized stiffness tensor (using 11 classical matrix notations [Walpole, 1984; François, 1995]) as a function of 12 the applied deformation (cells are loaded in tension in the bridges direction) 13 for a cell of size $r=5 \times 5$ RVEs. It is worth noting that the cell stiffness in 14 both the orthogonal (walls) direction (term $H_{22}$ ) and the shear (term $H_{33}$ ) 
are drastically reduced even if the cell is loaded uniaxially in the bridges

2 direction. This highlights the need to take into account the whole stiffness

3 tensor to accurately simulate the failure process as the stress redistribution

4 between neighboring cells is influenced by their local stiffness.

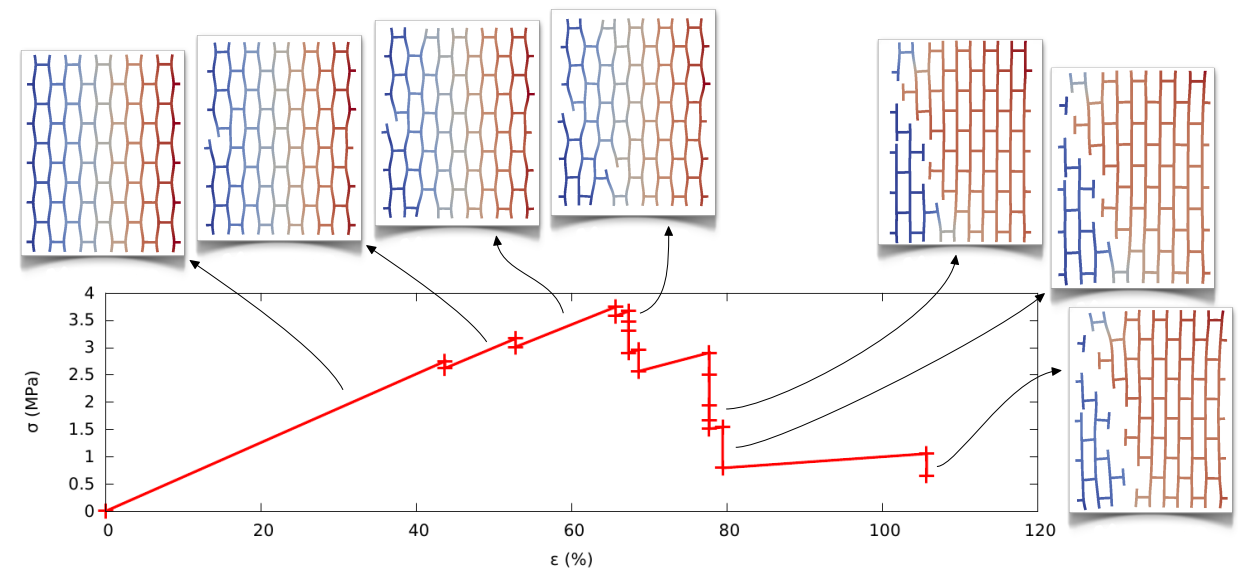

Figure 4: One run of the micromechanical model on a portion of scaffold (size $r=5 \times 5$ RVEs) under pure traction (plus periodicity conditions), showing the macroscopic stressstrain curve, and displacement fields over the deformed geometries for several reached states. Despite being very basic, the model is able to represent failure of both walls and bridges, eventually outside the main crack, which is not fully orthogonal to the macroscopic loading.

\section{3.2. Limitations of the weakest link theory}

6 Compared to the current method, Weibull's weakest link theory [Weibull, 7 1939] has many limitations in the scaling of strength distribution in quasi-

8 brittle systems. Basically, it presents a relationship between failure proba-

9 bilities at different volumes $V_{1}$ and $V_{2}$ as:

$$
p^{F}\left(V_{2}\right)=1-\left(1-p^{F}\left(V_{1}\right)\right)^{\frac{V_{2}}{V_{1}}}
$$




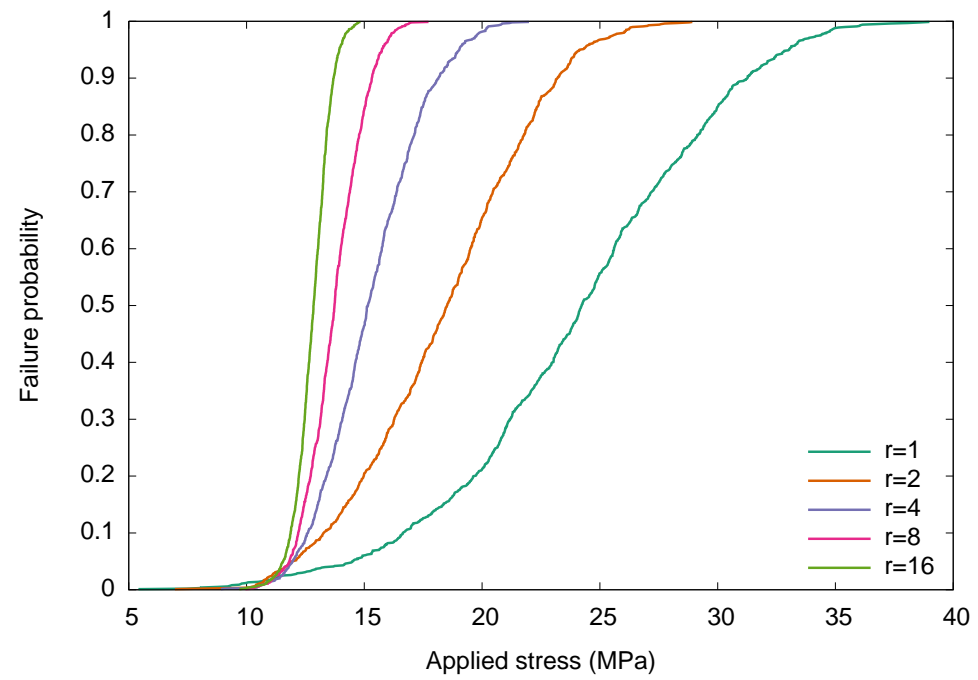

Figure 5: Strength distribution of successively larger micro-cells (size $r=1 \times 1,2 \times 2$, $4 \times 4,8 \times 8$ and $16 \times 16$ RVEs), highlighting the important size effect in such quasi-brittle structures.

1 Since this relies on the idea that the failure of a single element of volume 2 induces the failure of the whole structure (more precisely, if $V_{2}>V_{1}$, the 3 failure of an element of size $V_{1}$ induces the failure of the larger element of size $\left.4 V_{2}\right)$, it cannot be strictly applicable for quasi-brittle materials. This is illus5 trated in Figure 7, where for several micro-cells of increasing size $(r=2 \times 2$, $64 \times 4,8 \times 8$ and $16 \times 16$ RVEs), we compare their strength distribution com7 puted using the micromechanical model to the one obtained by scaling the 8 strength distribution of one RVE using Equation (2). Clearly, the weakest

9 link theory is only valid for the smaller sizes, which are actually brittle and 10 for which the exact and scaled strength distributions match perfectly. This 11 is not the case anymore for the larger sizes, for which several local failures 12 are required to trigger the global failure. 

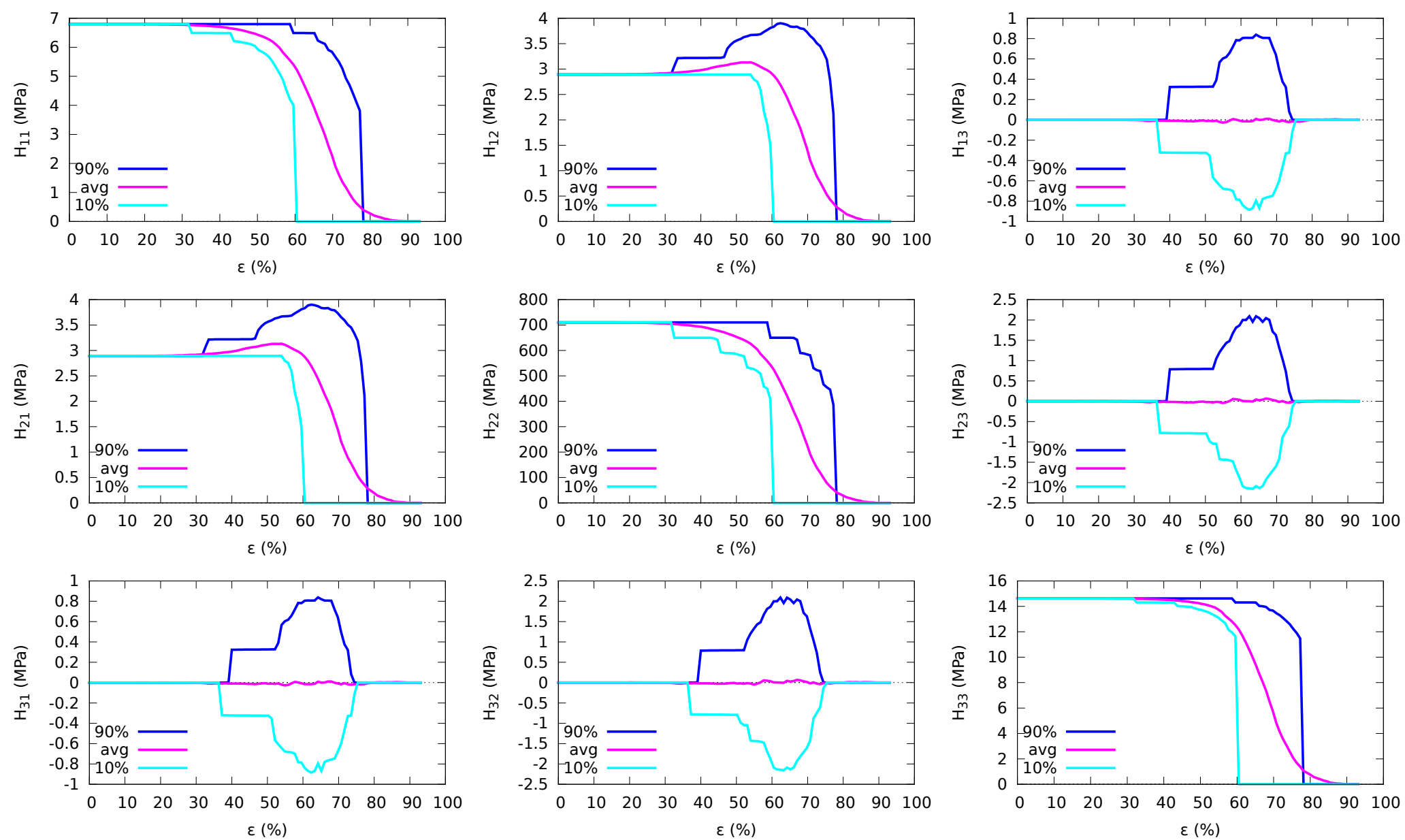

Figure 6: Distributions of homogenized stiffness tensor components (in matrix notations

[Walpole, 1984; François, 1995]) $\left(H_{i j}\right)$ as a function of applied strain $(\epsilon)$ on a cell of size $r=5 \times 5$ RVEs. Cells are loaded in tension in the bridges direction. Mean values, as well as $10 \%$ smallest and largest values, are show to highlight the dispersion. One can see the progressive reduction of mechanical properties associated to successive local failures. 

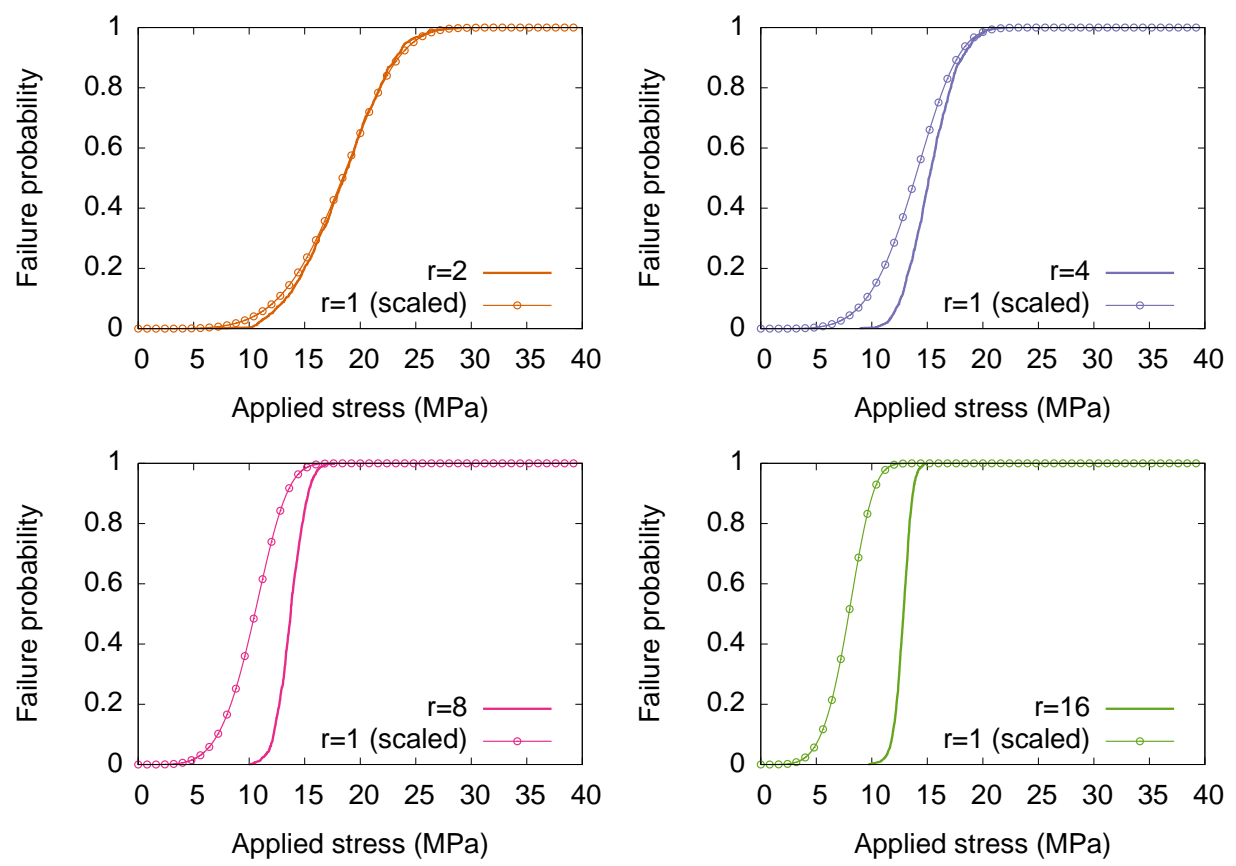

Figure 7: Comparison between the strength distributions obtained on one RVE and then scaled to a larger volume (circles), and the strength distributions directly obtained on larger micro-cells (solid lines). One can see that the weakest link scaling only applies for very small cells where behavior is brittle. 


\subsection{Validation of the computational homogenization-based scaling method} for strength distribution

In order to establish the proposed homogenized model, we compared its predictions to those obtained directly with the micromechanical model detailed in section 2.1. To do so, we created macroscopic meshes equivalent to the microscopic ones, where each micro-cell is replaced by a macro-cell of the same shape and size, but meshed with only a few triangular elements, as illustrated in Figure 8. Note that, in principle, a single quadrangle finite element could have been used for each macro-cell. We have checked that the discretization of the macro-cells did not have any effect on the macroscopic results. The resulting strength distributions are shown on Figure 9. The predictions based upon the homogenized model match almost perfectly the ones based on the micromechanical model, and this for small sizes (where the final failure is brittle) as well as for larger sizes (where the final failure is induced by many local failures). We also found that the failure patterns were visually similar between the micro- and macro-models. These results prove that it is sufficient to handle the stress redistribution between neighboring RVE in a homogenized manner. As each RVE exhibits a brittle failure triggered by the first bridge break, it could be chosen as the minimal failure volume in the structure. Therefore, only the average stress state over the

RVE has to be considered with respect to the RVE failure. Moreover, the stiffness reduction in the other directions is captured by the evaluation of the residual mechanical properties for each damage state.

\subsection{Application to failure prediction of macroscopic scale samples}

Based on the homogenized model established in this work, we can now scale the strength distributions obtained on a given micro-cell to virtually 


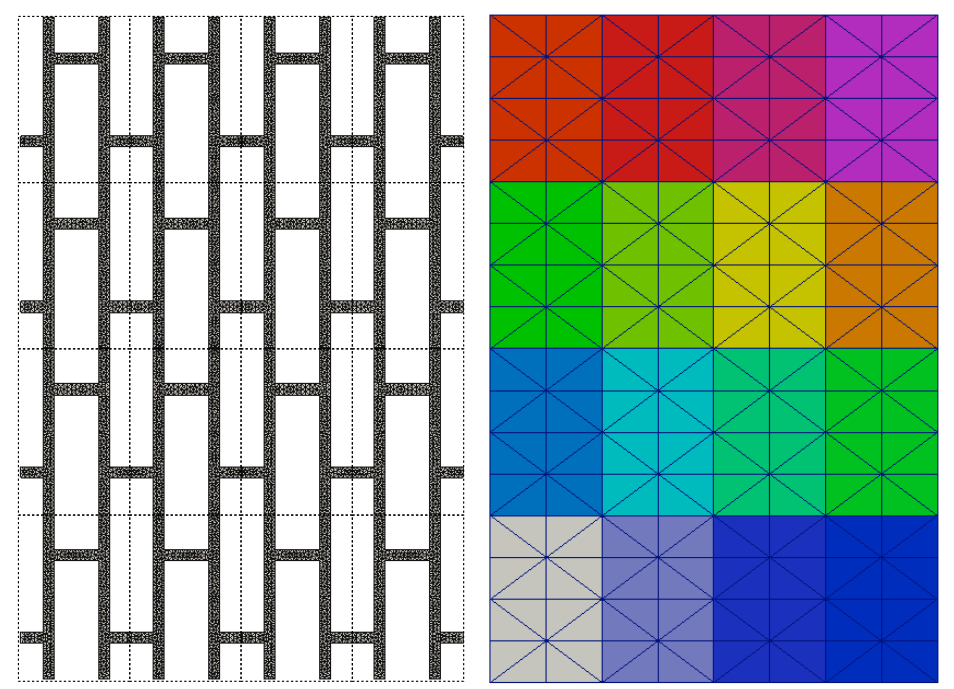

Figure 8: Finite element mesh of a micro-cell with $r=4 \times 4$ RVEs (left) and corresponding mesh used for the homogenized computations (right). Each RVE is replaced by a macrocell of the same size but meshed with only 16 triangular elements.

1 any size, thereby enabling the study of the shape and scaling properties of 2 the strength distributions across scales. The ceramic brick-like microstruc3 ture studied in the present work (Figure 1(a)) represents a complex system 4 with in-parallel (i.e., where local failures generate over-load on the neigh5 boring constituents) and in-series (i.e., where local failures also unload some 6 neighboring constituents) connections; as such its strength distribution can7 not be represented a priori by canonical distributions such as the Weibull 8 distribution (as is the case for in-series systems) or a normal distribution 9 (as is the case for large in-parallel systems [Daniels, 1945]). Similarly, as 10 evidenced by Figure 7, except for very small sample sizes the size effect on 11 the strength distributions cannot be described by a simple law such as the 12 power law (as is the case for solely in-series systems). However, it is pos13 sible to investigate the shape and scaling properties a posteriori from the 

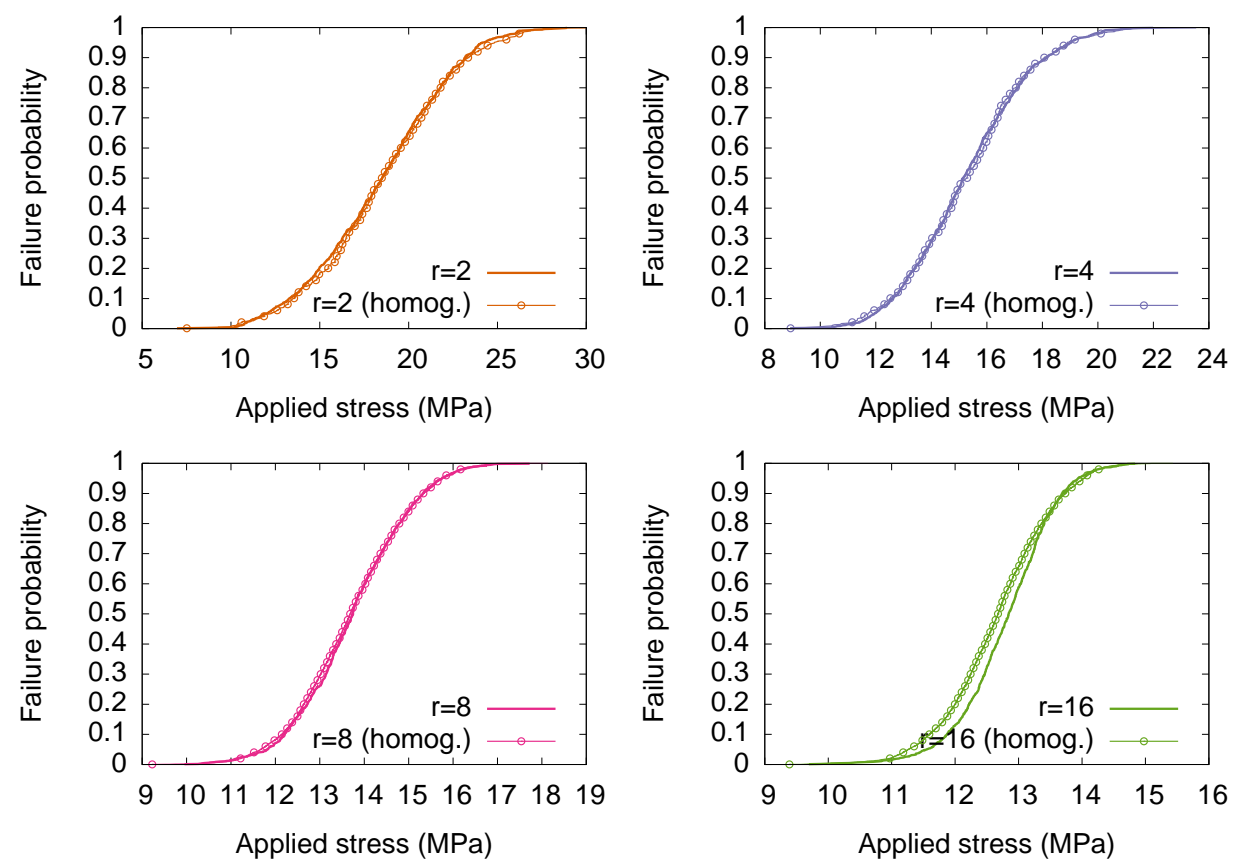

Figure 9: Comparison of strength distribution predicted by the micro model (continuous lines) and the homogenized model (circles) for microstructures of various size scales. This establishes the capability of the proposed homogenized model to predict the strength distribution of structures of virtually any size. 
1 numerical computations. For instance, Figure 10 illustrates the evolution

2 of the strength as a function of the size of the considered microstructure,

3 from micro to macro scales. One can recognize the size effect that has been 4 documented experimentally for quasi-brittle materials, e.g., [Bažant, 1999].

5 Since we compute the entire strength distributions for several sample

6 sizes, it is possible to study their shape and scaling relationships. Figure 11 7 shows the fit error (i.e., the distance between the set of points and the fitted 8 law), for both Weibull and normal distribution laws, as a function of the 9 sample size. One can distinguish three domains on the curves: (i) for very small samples, i.e., of the size of RVE, the strength distribution is of Weibull

1 shape, which is consistent with the hypothesis that walls and bridges failure 2 follows a Weibull law and that the brittle failure of the RVE is triggered by the first bridge break; (ii) there is an intermediate domain where strength distribution is closer to a normal distribution than to Weibull; (iii) finally, 15 for macroscopic scale samples, the strength distribution appears to revert 16 to a Weibull shape. Note that the parameters of the macroscopic Weibull 17 distribution differ from the ones of the RVE scale and cannot be predicted by simply scaling the RVE scale parameters. The particular relationship between the sets of parameters is indeed an outcome of our method. The 20 conclusions are admittedly linked to the particular system studied in this pa21 per and the hypothesis underlying the chosen micromodel, but our approach 22 does illustrate the prediction capabilities of the proposed method.

23 Not surprisingly, these findings are similar to the predictions of [Bažant 24 and Pang, 2007]. The only difference is that the initial strength distribution 25 is Weibull-shaped and not Gaussian, which is due to the modeling choices underlying the micromechanical model, especially the fracture model of the 27 material constituents. Here we assumed that the defects triggering failure 
1 are at a much lower length scale, so that the constituents failure is well de2 scribed by a Weibull law, which in turns generate a Weibull-shaped strength distribution for the geometrical RVE.

The above discussion is concerned only with the shape of the strength 5 distribution across scales, but scaling relationships can also be studied. Fig6 ure 13 shows the scaling error (i.e., the distance between the scaled strength 7 distribution and the reference one), supposing a weakest link scaling, as a 8 function of sample size for several cells of increasing size. The strength dis-

${ }_{26}(r=256 \times 256 \mathrm{RVEs})$. An initial step in the fracture process consists 27 of a widespread development of damage due to the uncorrelated failure of 
the weakest local defects. Stress redistribution caused by these failures is not significant enough to make the neighboring cells break or to initiate a macrocrack as the clusters of broken cells remain small with a typical size of $2-3$ cells. Eventually a critical defect is activated, rapidly leading to the development of an incipient macrocrack which leads to the final failure of the specimen. It is important to distinguish this type of "brittle-like" failure from a failure that would be induced by many correlated events. The "fatal" macrocrack does not result from the percolation of previously damaged cells; moreover, the location of the critical defect is not necessarily within the most damaged area of the specimen. It appears that two populations of defects can be identified: (i) a population of non-critical defects corresponding to the weakest local defects activated at a low stress level; and (ii) a population of critical defects, uncorrelated from the first ones, which can lead to the brittle failure of the specimen. Indeed, this represents another example why it is possible to predict the failure of similar materials based on an ad hoc description of the critical defects population, as previously shown by the authors [Genet et al., 2013a].

\section{Summary and Perspectives}

We have presented a multi-level numerical method which provides the means to derive reliable structure-strength relationships including statistical and size-dependent aspects, suitable to virtually any quasi-brittle material and any engineering component made from it. There are numerous potential applications with this methodology. Such models can be used by materials engineers to optimize fabrication processes to optimize their microstructures in a quantitative way; they can also be used by mechanical or civil engineers 


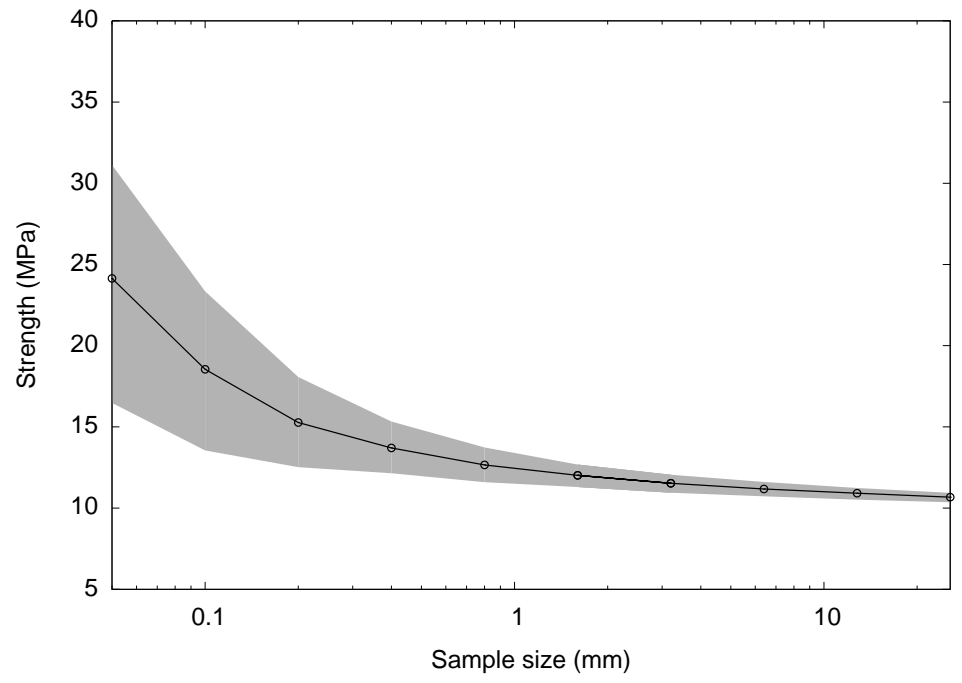

Figure 10: Strength associated with $50 \%$ failure probability (continuous line) and range of strength associated with failure probability between $10 \%$ and $90 \%$ (grey area), as a function of the structure size. The homogenized model based method proposed in this work permits the computation of a very wide range of sizes, from the micro to- the macro-scales. The computed behavior corresponds to what is found experimentally for quasi-brittle materials [Bažant, 1999]. 


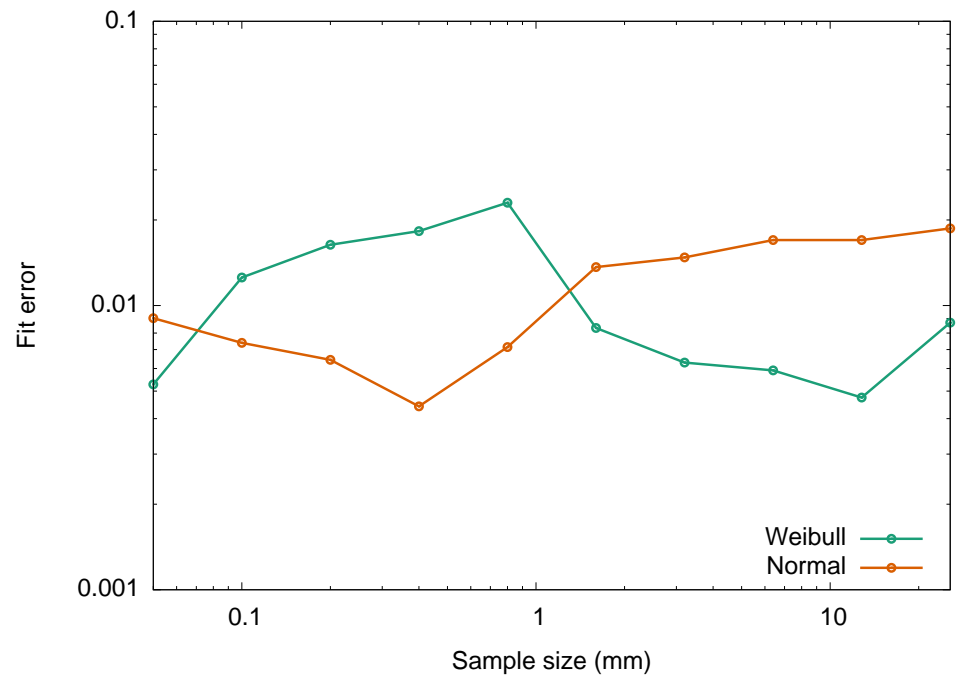

Figure 11: Fit error for both Weibull and normal distribution laws, as a function of the sample size. Strength distributions are of Weibull type for the RVE and macroscopic samples; they are closer to normal type for intermediate sample sizes.

1 to perform reliability analysis and derive optimum designs for specific ap2 plications.

3 The methodology also provides some fundamental insight to the failure of 4 quasi-brittle systems, a subject of widespread interest for many decades, but 5 rarely studied in its full complexity to include statistical and size-dependent 6 effects. With this approach, we were able to determine three domains of 7 failure patterns. Our most important conclusion is that the shape of the 8 strength distribution, after being closer to normal for intermediate scale 9 samples, reverts to Weibull for macroscopic scale samples. The Weibull 10 coefficients of the macroscopic law are different compared to the ones of 11 the microscopic law, and the link between the two sets of parameters is an 12 outcome of the method. This conclusion is in qualitative agreement with 13 Bažant's theory [Bažant and Pang, 2007]. An immediate perspective of this 


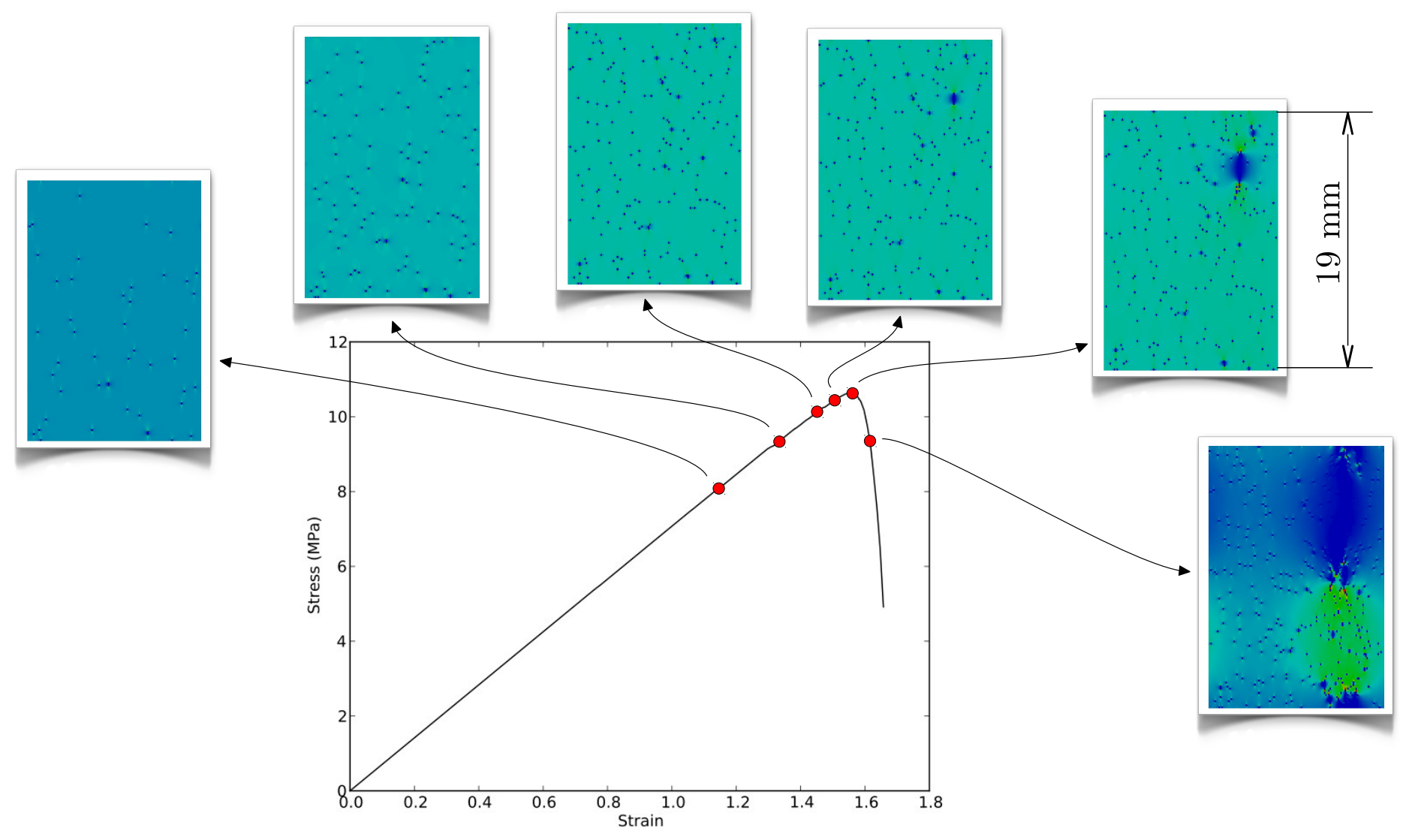

Figure 12: Stress-strain curve and stress field snapshots for one run of the homogenized model on a large scaffold (size $r=256 \times 256$ RVEs) under traction (plus periodicity conditions), with stress-strain curve and stress fields (dark blue zones correspond to zero level stress, i.e., broken RVEs) over the deformed geometries at multiple time points, showing that macroscopic failure is induced by the sudden activation of a macroscopic defect. 


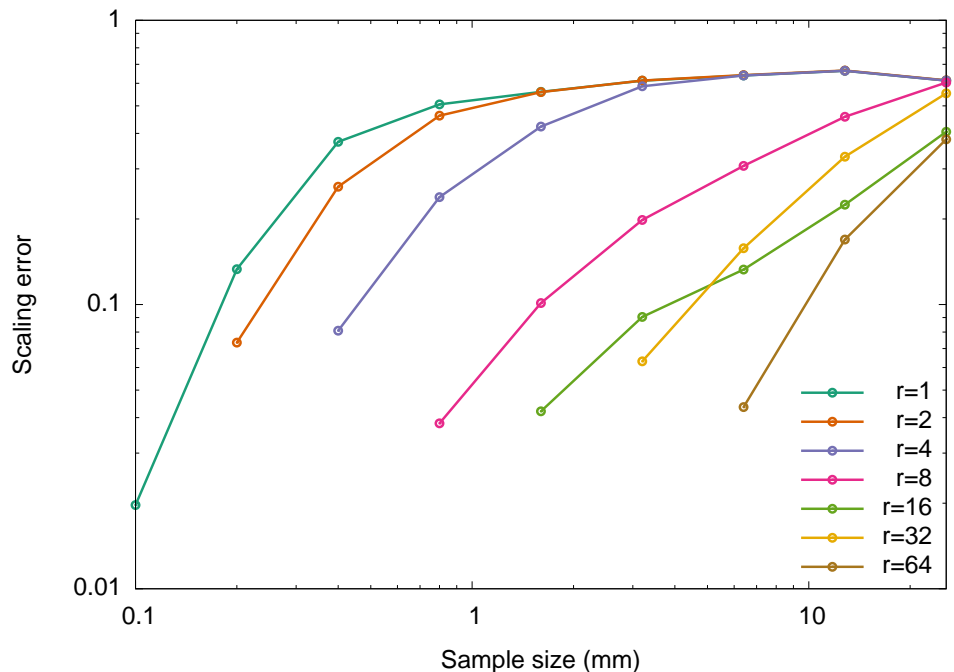

Figure 13: Scaling error for several cells of increasing size $r \times r$ as a function of the sample size considering a weakest link scaling law, showing that weakest link scaling only applies on the very small samples.

work will be to perform quantitative comparisons between our numerical predictions and this theory. However, it is important to point out that once the micromechanical model is defined, our approach does not require 4 any additional assumption to predict the scaling laws. Thus, the number 5 of "chains" and "bundles" of the equivalent hierarchical microstructure re6 quired in the Bažant model could be identified directly by our approach.

$7 \quad$ This capability of our approach allows us to use it to provide guidelines 8 for the processing of optimized Nature-inspired materials. Indeed, as our 9 intent here was to focus on the method itself, we used a simplistic microme10 chanical model, but we plan now to study the effect of varying microstruc11 tural parameters and the introduction of different toughening mechanisms 12 on the scaling laws of a given material. These variations will impact the 13 geometrical RVE failure distribution, as well as the length scales at which 


\section{References}

P. R. Amestoy, I. S. Duff, and J.-Y. L'Excellent. Multifrontal parallel distributed symmetric and unsymmetric solvers. Computer Methods in Applied Mechanics and Engineering, 184(2-4):501-520, April 2000. ISSN 00457825. DOI 10.1016/S0045-7825(99)00242-X. 
S. B. Batdorf and H. L. Heinisch. Weakest Link Theory Reformulated for Arbitrary Fracture Criterion. Journal of the American Ceramic Society, 61(7-8):355-358, July 1978. ISSN 0002-7820. DOI 10.1111/j.11512916.1978.tb09327.x.

Zdeněk P. Bažant. Size effect on structural strength : a review. Archive of Applied Mechanics, 69:703-725, 1999.

Zdeněk P. Bažant. Scaling theory for quasibrittle structural failure. Proceedings of the National Academy of Sciences of the United States of America, 101(37):13400-7, September 2004. ISSN 0027-8424. DOI 10.1073/pnas.0404096101.

Zdeněk P. Bažant and Sze-Dai Pang. Activation energy based extreme value statistics and size effect in brittle and quasibrittle fracture. Journal of the Mechanics and Physics of Solids, 55(1):91-131, January 2007. ISSN 00225096. DOI 10.1016/j.jmps.2006.05.007. URL http://linkinghub. elsevier.com/retrieve/pii/S0022509606001025.

Zdeněk P. Bažant and Yunping Xi. Statistical size effect in quasi-brittle structures: II. Nonlocal theory. Journal of Engineering Mechanics, 117 (11):2609-2622, 1991.

Zdeněk P. Bažant, Yunping Xi, and Stuart G. Reid. Statistical size effect in quasi-brittle structures: I. Is Weibull theory applicable? Journal of Engineering Mechanics, 117(11):2609-2622, November 1991. ISSN 07339399. DOI 10.1061/(ASCE)0733-9399(1991)117:11(2609).

Zdeněk P. Bažant, Jia-Liang Le, and Martin Z. Bazant. Scaling of strength and lifetime probability distributions of quasibrittle structures 
based on atomistic fracture mechanics. Proceedings of the National Academy of Sciences of the United States of America, 106(28):11484-9, July 2009. ISSN 1091-6490. DOI 10.1073/pnas.0904797106. URL http://www.pubmedcentral.nih.gov/articlerender.fcgi?artid= 2710678\&tool=pmcentrez\&rendertype=abstract .

Matthew R. Begley, Noah R. Philips, Brett G. Compton, David V. Wilbrink, Robert O. Ritchie, and Marcel Utz. Micromechanical models to guide the development of synthetic 'brick and mortar' composites. Journal of the Mechanics and Physics of Solids, 60(8):1545-1560, August 2012. ISSN 00225096. DOI 10.1016/j.jmps.2012.03.002.

Vincent Calard and Jacques Lamon. Failure of fiber bundles. Composites Science and Technology, 64(5):701-710, April 2004. ISSN 02663538. DOI 10.1016/j.compscitech.2003.07.003. URL www.scopus.com.

Yanqing Chen, Timothy A. Davis, William W. Hager, and Sivasankaran Rajamanickam. Algorithm 887: CHOLMOD, supernodal sparse Cholesky factorization and update/downdate. ACM Transactions on Mathematical Software, 35(3):1-14, October 2008. ISSN 00983500. DOI 10.1145/1391989.1391995.

Guillaume Couégnat. Multiscale approach of the mechanical behavior of composite materials with woven reinforcements (in French). Phd thesis, Bordeaux 1 University, 2008.

Guillaume Couégnat, W Ros, T Haurat, C Germain, Éric Martin, and G Vignoles. An integrated virtual material approach for ceramic matrix composites. Ceram. Eng. Sci. Proc., 33:83--91, 2013. DOI 10.1002/9781118217542.ch8. 
H L Cox. The elasticity and strength of paper and other fibrous materials. British Journal of Applied Physics, 3(3):72-79, March 1952. ISSN 05083443. DOI 10.1088/0508-3443/3/3/302.

H. E. Daniels. The Statistical Theory of the Strength of Bundles of Threads. I. Proceedings of the Royal Society A: Mathematical, Physical and Engineering Sciences, 183(995):405-435, June 1945. ISSN 1364-5021. DOI 10.1098/rspa.1945.0011.

René De Borst, J. Pamin, R. H. J. Peerlings, and L. J. Sluys. On gradientenhanced damage and plasticity models for failure in quasi-brittle and frictional materials. Computational Mechanics, 17(1-2):130-141, December 1995. ISSN 0178-7675. DOI 10.1007/BF00356485.

Rodrigue Desmorat, Fabrice Gatuingt, and Frédéric Ragueneau. Nonlocal anisotropic damage model and related computational aspects for quasibrittle materials. Engineering Fracture Mechanics, 74(10):1539-1560, July 2007. ISSN 00137944. DOI 10.1016/j.engfracmech.2006.09.012.

Sylvain Deville, Eduardo Saiz, Ravi K. Nalla, and Antoni P. Tomsia. Freezing as a path to build complex composites. Science, 311(5760):515-8, January 2006. ISSN 1095-9203. DOI 10.1126/science.1120937.

Anthony G. Evans. A General Approach for the Statistical Analysis of Multiaxial Fracture. Journal of the American Ceramic Society, 61(7-8):302-308, July 1978. ISSN 0002-7820. DOI 10.1111/j.1151-2916.1978.tb09314.x.

Christian Fagiano, Martin Genet, Emmanuel Baranger, and Pierre Ladevèze. Computational geometrical and mechanical modeling of woven 
ceramic composites at the mesoscale. Composite Structures (Accepted), 2014 .

Marc François. Identification des symetries materielles de materiaux anisotropes. PhD thesis, 1995.

A. M. Freudenthal. Statistical approach to brittle fracture. In H. Liebowitz, editor, Fracture, pages 591-619. Academic Press, 1968.

Martin Genet. Toward a virtual material for ceramic composites (in French). Phd thesis, ENS-Cachan, 2010.

Martin Genet, Lionel Marcin, Emmanuel Baranger, Christophe Cluzel, Pierre Ladevèze, and Anne Mouret. Computational prediction of the lifetime of self-healing CMC structures. Composites Part A: Applied Science and Manufacturing, 43(2):294-303, February 2012. ISSN 1359835X. DOI 10.1016/j.compositesa.2011.11.004.

Martin Genet, Manuel Houmard, Salvador Eslava, Eduardo Saiz, and Antoni P. Tomsia. A two-scale Weibull approach to the failure of porous ceramic structures made by robocasting: possibilities and limits. Journal of the European Ceramic Society, 33(4):679-688, April 2013a. ISSN 0955-2219. DOI 10.1016/j.jeurceramsoc.2012.11.001.

Martin Genet, Lionel Marcin, and P. Ladeveze. On structural computations until fracture based on an anisotropic and unilateral damage theory. International Journal of Damage Mechanics, September 2013b. ISSN 10567895. DOI 10.1177/1056789513500295. URL http://ijd.sagepub.com/ cgi/doi/10.1177/1056789513500295. 
Christophe Geuzaine and Jean-François Remacle. Gmsh: a threedimensional finite element mesh generator with built-in pre- and postprocessing facilities. International Journal for Numerical Methods in Engineering, 79(11):1309-1331, September 2009. ISSN 00295981. DOI 10.1002/nme.2579.

Lorna J. Gibson and Michael F. Ashby. Cellular Solids: Structure and Properties - Second Edition. Cambridge University Press - Cambridge Solid State Science Series, 1997. ISBN 0521499119. DOI 10.2277/0521499119.

Laurent Guillaumat and Jacques Lamon. Probabilistic-statistical simulation of the non-linear mechanical behavior of a woven $\mathrm{SiC} / \mathrm{SiC}$ composite. Composites Science and Technology, 56:803-808, 1996.

François Hild. Damage, Failure and Scale Bridging in Heterogeneous Materials (in French). Habilitation, ENS-Cachan, 1998.

François Hild. The Weibull law: A model of wide applicability. In Proceedings of the NATO Advanced-Study-Institute on Physical Aspects of Fracture, volume 32, pages 35-46, 2001.

Manuel Houmard, Qiang Fu, Martin Genet, Eduardo Saiz, and Antoni P. Tomsia. On the structural, mechanical, and biodegradation properties of HA/ $\beta$-TCP robocast scaffolds. Journal of Biomedical Materials Research. Part B, Applied Biomaterials, 101(7):1233-42, October 2013. ISSN 15524981. DOI 10.1002/jbm.b.32935.

D. Jeulin. Morphological characterization and modeling of random structures (in French). In M. Bornert, T. Bretheau, and P. Gilormini, editors, 
Homogenization in Mechanics of Materials 1: Random Elastic Materials and Periodic Media (in French), pages 95-132. Hermès edition, 2001.

Baohua Ji and Huajian Gao. Mechanical properties of nanostructure of biological materials. Journal of the Mechanics and Physics of Solids, 52(9):1963-1990, September 2004. ISSN 00225096. DOI 10.1016/j.jmps.2004.03.006.

Jacques Lamon. Stochastic approach to multiple cracking in composite systems based on the extreme-values theory. Composites Science and Technology, 69(10):1607-1614, August 2009. ISSN 02663538. DOI 10.1016/j.compscitech.2009.03.009.

Maximilien E. Launey, Etienne Munch, D. H. Alsem, H. B. Barth, Eduardo Saiz, Antoni P. Tomsia, and Robert O. Ritchie. Designing highly toughened hybrid composites through nature-inspired hierarchical complexity. Acta Materialia, 57(10):2919-2932, June 2009. ISSN 13596454. DOI 10.1016/j.actamat.2009.03.003.

Jia-Liang Le and Zdeněk P. Bažant. Unified nano-mechanics based probabilistic theory of quasibrittle and brittle structures: II. Fatigue crack growth, lifetime and scaling. Journal of the Mechanics and Physics of Solids, 59(7):1322-1337, July 2011. ISSN 00225096. DOI 10.1016/j.jmps.2011.03.007. URL http://linkinghub.elsevier.com/ retrieve/pii/S0022509611000524.

Jia-Liang Le, Zdeněk P. Bažant, and Martin Z. Bazant. Unified nanomechanics based probabilistic theory of quasibrittle and brittle structures: I. Strength, static crack growth, lifetime and scaling. Journal of the Mechanics and Physics of Solids, 59(7):1291-1321, July 2011. ISSN 
00225096. DOI 10.1016/j.jmps.2011.03.002. URL http://linkinghub. elsevier.com/retrieve/pii/S0022509611000470.

Hugo Leclerc. Towards a no compromise approach between modularity, versatility and execution speed for computational mechanics on CPUs and GPUs. In Olivier Allix and Peter Wriggers, editors, IV European Conference on Computational Mechanics (ECCM2010), Paris, France, 2010.

Jean Lemaître and Rodrigue Desmorat. Engineering Damage Mechanics: Ductile, Creep, Fatigue and Brittle Failures. Springer, 2005.

Jean Lemaître, Jean-Louis Chaboche, Rodrigue Desmorat, and Ahmed Benallal. Solid Materials Mechanics, Third Edition (In French). Dunod, 2009 .

Jacky Mazars and Gilles Pijaudier-Cabot. Continuum damage theoryApplication to concrete. Journal of Engineering Mechanics, 115(2):345$365,1989$.

Etienne Munch, Maximilien E. Launey, D. H. Alsem, Eduardo Saiz, Antoni P. Tomsia, and Robert O. Ritchie. Tough, bio-inspired hybrid materials. Science, 322(5907):1516-20, December 2008. ISSN 1095-9203. DOI 10.1126/science.1164865.

Valentina Naglieri, Hrishikesh A. Bale, Bernd Gludovatz, Antoni P. Tomsia, and Robert O. Ritchie. On the development of ice-templated silicon carbide scaffolds for nature-inspired structural materials. Acta Materialia, 61(18):6948-6957, October 2013. ISSN 13596454. DOI 10.1016/j.actamat.2013.08.006. 
S. Leigh Phoenix. Probabilistic strength analysis of fibre bundle structures. Fibre Science and Technology, 7:15-31, 1974. URL http://www. sciencedirect.com/science/article/pii/0015056874900037.

S. Leigh Phoenix. Stochastic strength and fatigue of fiber bundles. International Journal of Fracture, 14:327-344, 1978. URL http://link. springer.com/article/10.1007/BF00034692.

S. Torquato. Random Heterogeneous Materials: Microstructure and Macroscopic Properties. Springer edition, 2002.

L. J. Walpole. Fourth-Rank Tensors of the Thirty-Two Crystal Classes: Multiplication Tables, 1984. ISSN 1364-5021.

Wallodi Weibull. A statistical theory of the strength of materials. Royal Swedish Institute for Engineering Research, 151, 1939.

Wallodi Weibull. A statistical distribution function of wide applicability. Journal of applied mechanics, pages 293-297, September 1951. 